DOI 10.31489/2019No2/74-77

UDC 621.7

\title{
STUDY OF THE RHEOLOGICAL PROPERTIES OF THE PLASTICIZER TO OBTAIN A COAL-WATER SLURRY
}

\author{
Tanasheva N.K. ${ }^{1}$, Minkov L.L. ${ }^{2}$, Tleubergenova A.Zh. ${ }^{3}$, Sadenova K.K. ${ }^{3}$ \\ ${ }^{1}$ Institute of Applied Mathematics, Karaganda, Kazakhstan \\ 2Tomsk State University, Tomsk, Russia \\ ${ }^{3}$ E. A. Buketov Karaganda State University, Karaganda, Kazakhstan, shymkent.a7@mail.ru
}

\begin{abstract}
The article presents the results of the study of the impact of plasticizers on the stability of coalwater suspensions obtained from Shubarkul coal slurries. As part of the task to determine the optimal reagent-plasticizer, an experimental installationwas developed. Fuel oil, gelatin, sodium humate were used as reagents-plasticizers. The process of enrichment of coal slurries of Shubarkul Deposit was studied; it is shown that the best results are obtained with the use of sodium humate. The use of sodium humate as a plasticizer makes it possible to create coal-water suspensions with a spatial network-like structure that do not delaminate for a long time.
\end{abstract}

Keywords: electro-hydro-impulse installation, Shubarkul coal, coal-water fuel, plasticizer reagent, ultrasonic dispersant.

\section{Introduction}

In the first half of the XXI century, it is to predict to increase the role of coal in the energy sector, due to its large reserves and depletion of oil and gas. At the same time, environmental problems arising from the use of coal fuel require the development and implementation of new economically and environmentally efficient coal technologies that will provide a significant environmental effect with the highest possible completeness of the use of the extracted fuel. Around many coal mining and coal processing enterprises, a large amount of extracted coal is accumulated in hydraulic dumps and sedimentation tanks, presented in the form of fine coal slurries, the transfer of which into a technologically acceptable fuel will not only improve the environmental situation in the regions, but also obtain a significant economic effect.

In this regard, it becomes relevant to use slurries in the form of coal-water suspensions (CWS), the development of effective processes for the production and application of which should be based on science-based processes of physical and physical-chemical effects on the source coal, taking into account the properties of its organic and mineral components [1]. CWS are mixtures of crushed coal with water. To give the suspension the properties of stability and the necessary fluidity, a small amount of reagent-plasticizer is introduced into the suspension. As a result, an artificial dispersion system is formed, representing a new type of fuel from coal - coal-water fuel (CWF).

The advantages of CWS as an ecological clean fuel are as follows:

- explosion prevention and fire safety in all technological operations (preparation, transportation, storage and use);

- absence of dust and pollution during storage and transportation; reduction of harmful emissions of nitrogen oxides, carbon and sulfur into the atmosphere during combustion.

In addition, CWS provide safety of technological properties during storage and transportation. At the same time, the decline in oil and gas production and the increase in their prices on the world and domestic markets in recent years have caused interest in CWF - a real alternative to liquid and gaseous organic type fuels.

The aim of this work is to study the rheological properties of reagents for stabilization of CWS. The object of study in this work is the coal of Shubarkul Deposit. 


\section{Experimental procedure}

The essence of the processes of preparation of Shubarkul coal slurries for use in CWS consists in their electro-hydro-impulse (EHI) treatment and further enrichment by the method of ultrasonic (US) agglomeration (since other methods of enrichment are not acceptable due to the low selectivity of these processes during enrichment due to the fine state of coal particles). Electric discharges in the liquid, which are a source of shock waves, are used to grind particles of coal slurries from the Shubarkul Deposit and obtain finely ground fractions of specified sizes. Shock waves propagating in the medium of liquid-solid coal particles destroy and grind the treated coals to small fractions, necessary for obtaining coal-water fuel.

In order to select the most effective plasticizers for CWS prepared from coal slurries, the influence of the following plasticizers was studied: gelatin, fuel oil, sodium humate obtained from coal. These reagents meet the requirements for plasticizing reagents and are readily available. The effectiveness of these additives is due to their physical and chemical properties, the peculiarity of their structure, consisting of hydrocarbon, aromatic, carboxyl, hydroxyl and other groups [2].

For experimental works on obtaining CWF in the laboratory of physics of pulsed phenomena in heterogeneous media of the Department of Engineering Thermophysics named after professor Zh.S. Akylbayev, electrohydropulse installation with the ultrasonic flow disperser was collected (Fig.1).

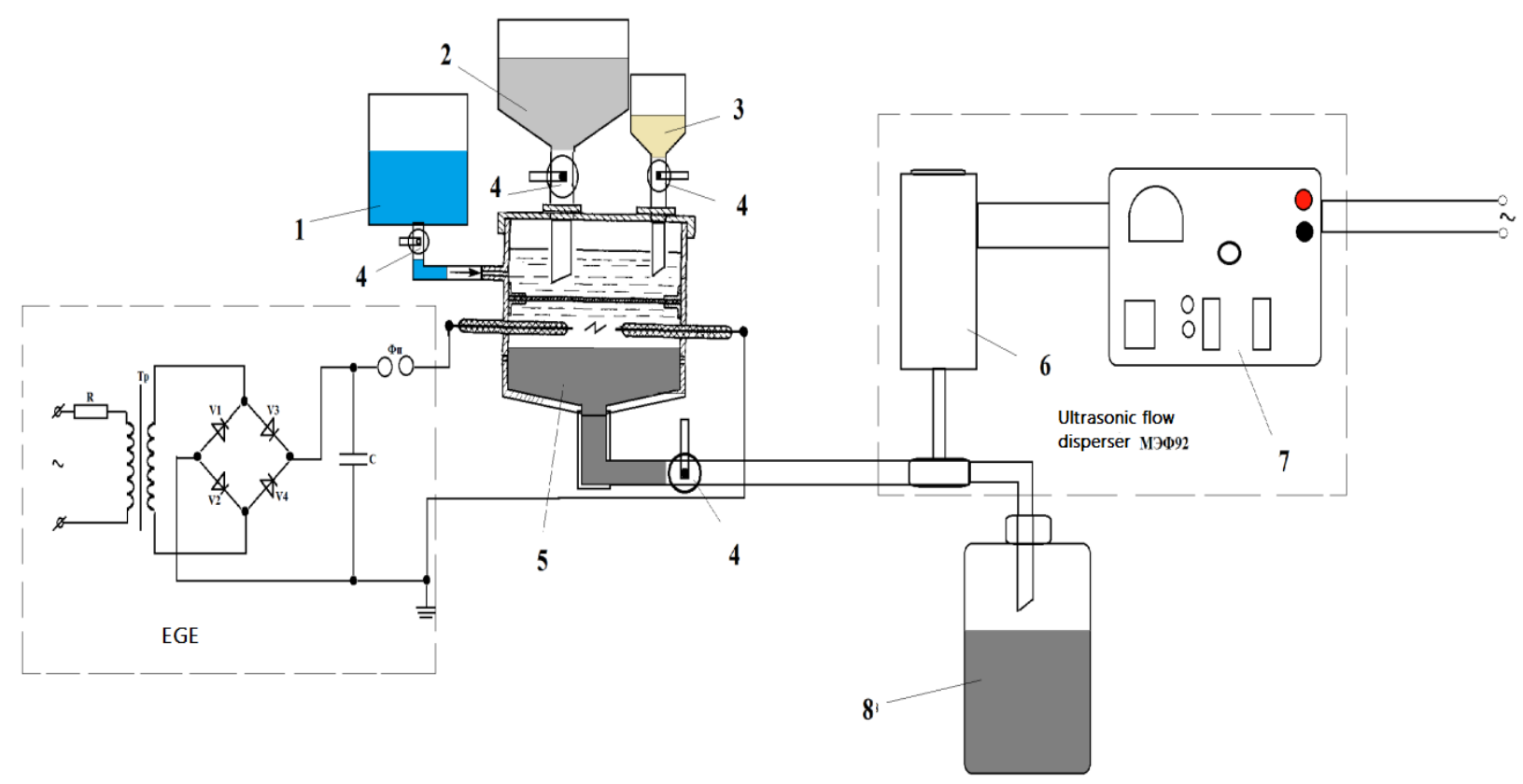

Fig.1. Schematic scheme of EHI installation with ultrasonic flow disperser:

1 - water tank, 2 - capacity for coal, 3 - tank for reagent - plasticizer, 4 -a valve for regulating, 5 - working cell with two opposite electrodes, 6 - ultrasonic flow transducer, 7 - the control unit of the dispersant, 8 - tank with WCF.

To create electro-hydraulic shocks, a scheme that includes a power source with a capacitor as a storage of electrical energy, is assembled. The voltage on the capacitor rises to a value at which there is a spontaneous breakdown of the air forming gap, and all the energy stored in the capacitor instantly enters the working liquid gap, where it is released in the form of a brief electric pulse of high power. Further, the process is repeated at a given capacitance and voltage with a frequency depending on the power of the supply transformer and the electrical and physical characteristics of the medium in the inter electrode gap [3]. 
The EHI installation works as follows, the prepared coal slurries were sent to the working cell, where there was an underwater electrical explosion in the presence of a plasticizer. As a result of EHI treatment coal-water suspension sent to the enrichment installation, the principle of operation of which was based on the method of ultrasonic agglomeration. The ultrasonic dispersant consists of a flow transducer and a control unit. The dispersant performs the function of additional crushing, where a chemical bond is formed between resulting suspensions. The resulting fuel is drained into the tank.

\section{Experimental results}

As a result of the performed enrichment studies, graphical dependences were obtained, which are presented in Fig.2-4.

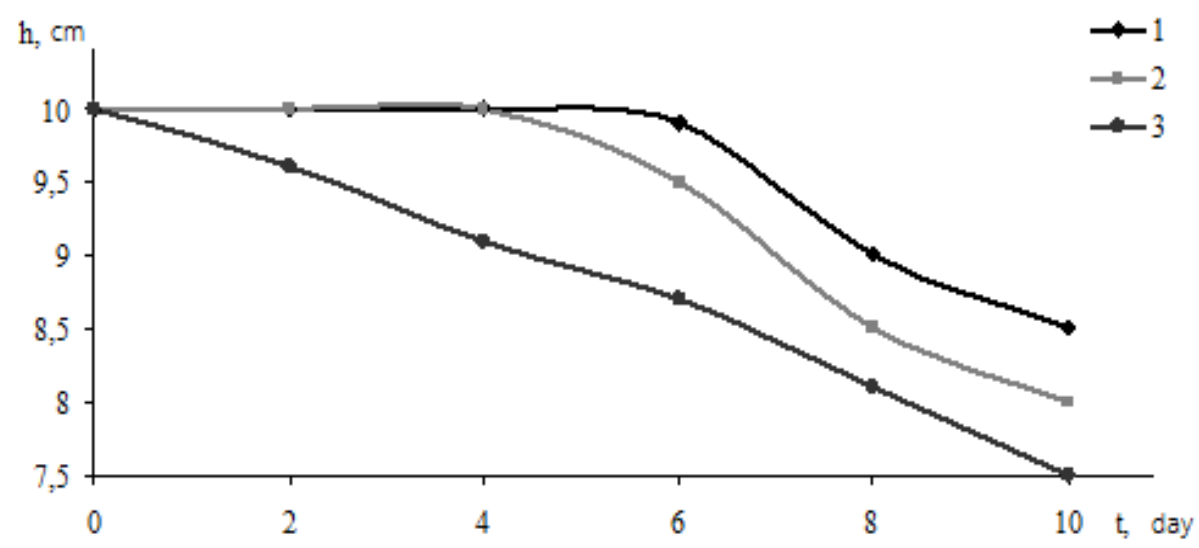

Fig.2. Dependence of the height of the dispersed phase layer on time (suspensions with additives of fuel oil of various quantity):

$1-1 \%$ to weight of coal; $2-0.5 \%$ to weight of coal; $3-2.0 \%$ to weight of coal

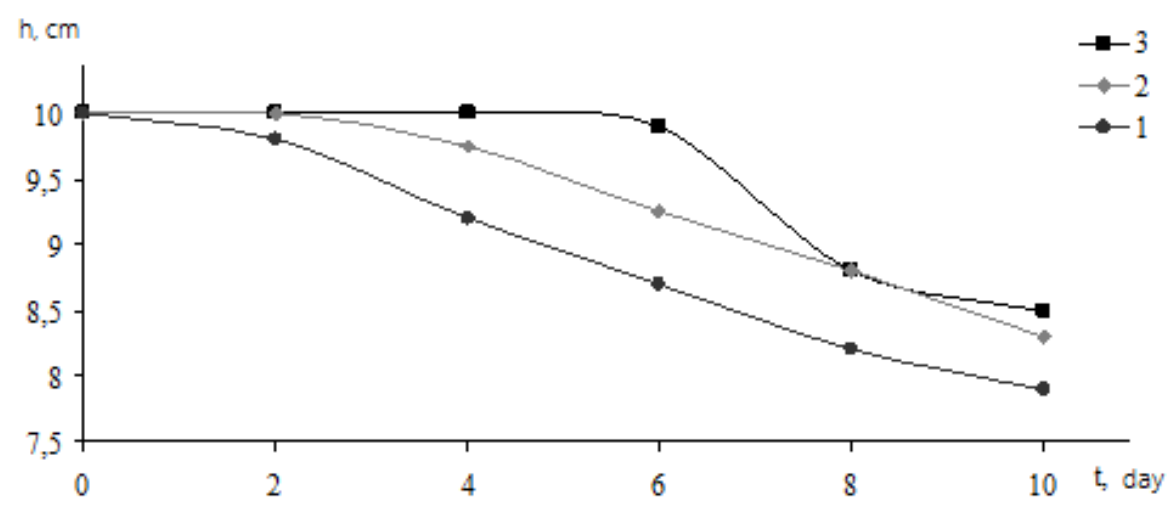

Fig.3. Dependence of the height of the dispersed phase layer on time (suspensions with gelatin additives of various quantity):

$1-0.5 \%$ to weight of coal; $2-1.0 \%$ to weight of coal; $3-2.0 \%$ to weight of coal

Organic compounds and technology of metered addition of the found compounds to the mass of CWF are selected. The process of formation of spatial structure of CWS is investigated, their rheological properties are studied, it is established that stability of CWS, received on the basis of concentrates, is defined by physical and chemical properties of plasticizers. It should be noted that suspensions in the presence of additives have good fluidity. From the graph it can be seen that with the addition of fuel oil in the amount of $1 \%$, the CWS had stability for a long time. 
Suspensions with the addition of gelatin (Fig.3) from $2 \%$ to weight of coal had stability for a long time, however, when the gelatin content is less than $1.0 \%$ to weight of Shubarkul coal, there is a noticeable decrease in the stability of suspensions.

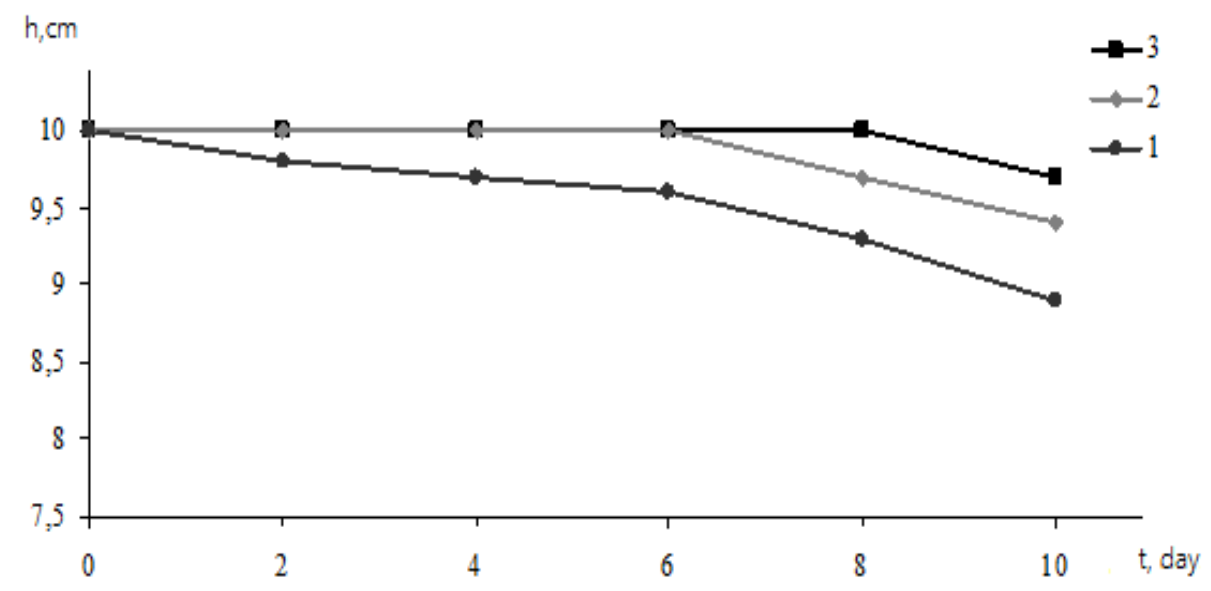

Fig.4. Dependence of the height of the dispersed phase layer on time (suspensions with various quantity of sodium humate additives):

$1-0.5 \%$ to weight of coal; $2-2.0 \%$ to weight of coal; $3-1.0 \%$ to weight of coal

Application as a stabilizer of humic preparation (Fig.4) (sodium humate) made it possible to obtain a stable CWS with stable and practically unchanged properties in time. The stability of CWS was maximal when using from $1 \%$ sodium humate to coal weight. After a very long storage, CWS were compacted to form loose sediments. When applying mechanical action (mixing) was the restoration of the original structure of the suspension.

\section{Conclusion}

Thus, on the basis of the results of the study, it was found that the addition of a stabilizersodium humate in the amount of $1 \%$ of the mass of coal-oil granules is due to the fact that at this rate, CWF has better stability. It is established that the stability of CWS obtained on the basis of coal-oil concentrates is determined by the physical and chemical properties of the reagentsplasticizers.

\section{Acknowledgment}

This work was financially supported by the RFBR project Mol_nr 19-38-50058

\section{REFERENCES}

1 Khodakov G.S. Suspension coal fuel (modern stage of research, technologies and industrial implementations). Izvestiya Akademii Nauk. Energy. 2000, No.2, pp.104 - 119. [in Russian]

2 Krapchin I.P. Economic efficiency of preparation and use of coal-water suspensionsenvironmentally friendly fuel for power plants. Coal. 2003, No.11, pp. $50-52$.

3 Kussaiynov K., Tanasheva N.K., Tolynbekov A., Alpysova G., Tleubergenova A. The research of burning technology of water coal fuel got from Shubarkul coal. Eurasian Physical Technical Journal. 2014, Vol.11, No. 1(21), pp. 7 - 11. 